\title{
Jointly Optimal Quantization, Estimation, and Control of Hidden Markov Chains ${ }^{1}$
}

\author{
John S. Baras, Xiaobo Tan, and Wei Xi \\ Institute for Systems Research and \\ Department of Electrical and Computer Engineering \\ University of Maryland, College Park, MD 20742 USA \\ $\{$ baras, xbtan, wxi\}@glue.umd.edu
}

\begin{abstract}
It is of interest to understand the tradeoff between the communication resource comsumption and the achievable system performance in networked control systems. In this paper we explore a general framework for tradeoff analysis and decision making in such systems by studying joint quantization, estimation, and control of a hidden Markov chain. Dynamic programming is used to find the optimal quantization and control scheme that minimizes a weighted combination of different cost terms including the communication cost, the delay, the estimation error, and the running cost. Simulation and analysis based on example problems show that this approach is able to capture the tradeoffs among competing objectives by adjusting the cost weights.
\end{abstract}

\section{Introduction}

Networked control systems have (potential) applications in defense, transportation, scientific exploration, and industry, with examples ranging from automated highway systems to unmanned aerial vehicles to MEMS sensor and actuator networks. Communication in networked control systems is often limited due to the large number of subsystems involved, limited battery life and power, and constraints imposed by environmental conditions. Hence an important concern in the development of networked control systems is how to deploy and allocate the communication resources. Proper understanding of the tradeoff between the communication resource consumption and the system performance will help to make such decisions. A great deal of effort has been put into the studies of control systems with communication constraints. In particular, stabilization of linear systems with quantized state/output/input has received much attention (see e.g., $[1,2,3]$ and the references therein). Estimation and control under commu-

\footnotetext{
${ }^{1}$ This research was supported by the Army Research Office under the ODDR\&E MURI01 Program Grant No. DAAD1901-1-0465 to the Center for Networked Communicating Control Systems (through Boston University), and under ARO Grant No. DAAD190210319.
}

nication constraints have also been studied $[4,5,6,7]$.

In this paper we explore a general framework for tradeoff analysis and decision making in networked control systems, by studying jointly optimal quantization, estimation, and control of a Hidden Markov chain. Hidden Markov chains form an important family of Hidden Markov Models (HMMs) [8], and have been widely used in speech processing, computer vision, computational biology, telecommunications, etc. Another reason for us to choose a hidden Markov chain is that numerical and even analytical solutions can be obtained relatively easily, which provides insight into the approach.

Fig. 1 illustrates the problem setup, $X_{n}$ is the state of a homogeneous, controlled, hidden Markov chain taking values in $\mathcal{X}=\left\{x_{1}, \cdots, x_{S}\right\}$ for some $S \geq 1$. The control $U_{n}$ takes values in $\mathcal{U}=\left\{u_{1}, \cdots, u_{K}\right\}$ for some $K \geq 1$, and the output $Y_{n}$ takes values in $\mathcal{Y}=\left\{y_{1}, \cdots, y_{M}\right\}$ for some $M \geq 1$. For $u \in \mathcal{U}$, $1 \leq i, j \leq S, 1 \leq k \leq M$ we denote

$$
\begin{gathered}
a_{i j}(u) \triangleq \operatorname{Pr}\left[X_{n+1}=x_{i} \mid X_{n}=x_{j}, U_{n}=u\right] \\
=\quad \operatorname{Pr}\left[X_{n+1}=x_{i} \mid X_{n}=x_{j}, U_{n}=u, X_{0}^{n-1}, U_{0}^{n-1}\right], \\
c_{i k} \triangleq \operatorname{Pr}\left[Y_{n}=y_{k} \mid X_{n}=x_{i}\right] \\
=\operatorname{Pr}\left[Y_{n}=y_{k} \mid X_{n}=x_{i}, X_{0}^{n-1}, U_{0}^{n}, Y_{0}^{n-1}\right],
\end{gathered}
$$

where the notation $Z_{n_{1}}^{n_{2}}$ denotes the sequence of random variables $\left\{Z_{n_{1}}, Z_{n_{1}+1}, \cdots, Z_{n_{2}}\right\}$. The quantized information $q_{n}$ of the output is sent over a communication channel to a remote processor, where state estimation and control computation are performed (later on we shall justify the "separation" of estimation from control in Fig. 1). The control $U_{n}$ is then sent back through a communication channel to the HMM. To highlight the main ideas and simplify the analysis, we assume that the communication is noise free.

This paper is divided into two parts. In the first part we are concerned only with joint quantization and estimation, i.e., the loop in Fig. 1 is not closed. Sequential vector quantization of Markov sources was consid- 


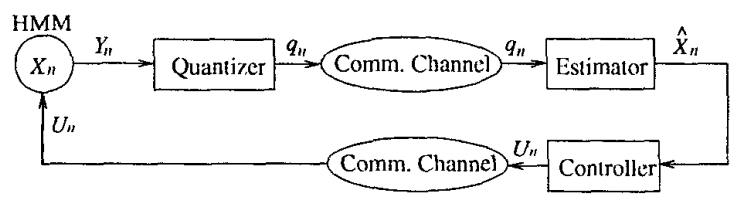

Fig. 1: The setup for joint quantization, estimation, and control of an HMM.

ered in [9], where a weighted combination of the entropy rate of the quantized process and the compression error was minimized. Such a "Lagrangian distortion measure" appeared earlier in [10]. A similar approach for combined classification and compression .was proposed in [11]. We extend the work in [9] to the case of vector quantization with variable block length, and seek the optimal quantization scheme to minimize a weighted combination of the estimation error, the entropy of the quantized output, and the delay due to block coding. The problem is recast as a stochastic control problem and the corresponding value function satisfies a Dynamic Programming (DP) equation of a novel form. The DP equation is solved numerically and the effects of weighting coefficients on optimal quantization schemes are studied through simulation.

In the second part of the paper the problem of joint quantization and control is discussed. Following the same spirit as in joint quantization and estimation, we seek the optimal quantization and control scheme to minimize a weighted sum of the communication cost and the cost relating to the system performance (the authors recently learned that a related work was reported in [7]). For illustrative purposes an example problem is solved analytically, which provides interesting insight into the approach.

In both the joint quantization/estimation problem and the joint quantization/control problem, the separation principle [12] holds. Either problem is decomposed into an estimation problem, and a decision (quantization/control) problem based on the state estimation.

The structure of the paper is as follows. In Section 2 the joint quantization and estimation problem is formulated and solved. Numerical solution of the DP equation is discussed and simulation results reported in Section 3. The joint quantization and control problem is studied in Section 4. Conclusions are provided in Section 5.

\section{Joint Quantization and Estimation}

\subsection{Problem formulation}

Vector quantization with variable block length is considered. Let $B \geq 1$ be the maximum block length. Given the a priori information about $X_{0}$, one deter- mines the length of the first data block $Y_{1}^{n_{1}}\left(n_{1} \leq B\right)$ and the quantization scheme for $Y_{1}^{n_{1}}$. At time $n_{1}$ the quantized $Y_{1}^{n_{1}}$ is sent, and one needs to determine the next data block $Y_{n_{1}+1}^{n_{2}}\left(n_{1}+1 \leq n_{2} \leq n_{1}+B\right)$ and the associated quantization scheme based only on the information available to the receiver (i.e., the information about $X_{0}$ and the quantized $Y_{1}^{n_{1}}$ ). This process goes on until the final time $N \geq 1$ is reached. Time instants (e.g., $0, n_{1}$ in the previous discussion) when one makes decisions are called decision times. Each transmission is assumed to complete instantly and the delay due to communication is assumed to be zero.

To formulate the problem precisely, let $\Omega_{1}$ be the space of admissible quantization decisions for $Y_{1}^{N}$. Here by a quantization decision, we mean a scheme for both division of $Y_{1}^{N}$ into (variable-length) blocks and quantization of these blocks. A quantization decision is admissible if at each decision time, the length of the next data block and the corresponding quantization scheme are decided based solely on the information available to the remote processor by that time. This requirement, as adopted in [9], is an "equimemory" (for the encoder and the decoder) condition [7]. It makes the sender's decision transparent to the receiver, and eliminates the need to transmit the quantization scheme separately. On the other hand this imposes the requirement of certain computation capability on the sender side, which, in some cases, is not feasible.

Let $\Pi_{0}=\left(\pi_{0}\left(x_{1}\right), \cdots, \pi_{0}\left(x_{S}\right)\right)$ be the a priori PMF (probability mass function) for $X_{0}$, where $\pi_{0}\left(x_{i}\right)=$ $\operatorname{Pr}\left[X_{0}=x_{i}\right], 1 \leq i \leq S$. Given $\Pi_{0}$ and $\omega \in \Omega_{1}$, define the cost

$$
J\left(\Pi_{0}, \omega\right)=E\left[\sum_{n=1}^{N} J^{q}(n)+\lambda_{d} J^{d}(n)+\lambda_{e} J^{e}(n)\right] .
$$

Here $\lambda_{d}, \lambda_{e} \geq 0$ are weighting coefficients, and $J^{q}(n)$, $J^{d}(n), J^{e}(n)$ are the cost terms relating to the communication needs, the delay due to block coding, and the estimation error at time $n$, respectively:

(1) $J^{q}(n)$ is the communication cost at time $n$. In this section we assume that entropy coding [13] is used, so the (expected) number of bits required to transmit a random vector $Z$ is bounded by $H[Z]+1$, where $H[Z]$ denotes the entropy of $Z$. Hence

$$
J^{q}(n)=\left\{\begin{array}{l}
0, \quad \text { if no transmission at time } n \\
H\left[Q_{n} \mid R_{n}\right]+1, \quad \text { otherwise }
\end{array}\right.
$$

where $Q_{n}$ represents the bits transmitted at time $n$, $R_{n}$ represents the bits sent before time $n$, and $H[\cdot \mid \cdot]$ denotes the conditional entropy;

(2) $J^{d}(n)$ is the delay cost evaluated at time $n$. For simplicity, we assume that $J^{d}(n)$ is equal to the number of samples being delayed at time $n$. For instance, if one decided to quantize $Y_{i-1}^{i+1}$ as a block, then $J^{d}(i-1)=1$ 
(since information about $Y_{i-1}$ has not been transmitted at time $i-1$ ), $J^{d}(i)=2$ (since information about both $Y_{i-1}$ and $Y_{i}$ has not been transmitted at time $\left.i\right), J^{d}(i+$ 1) $=0$ (no backlog at time $i+1)$;

(3) $J^{e}(n)$ is the cost reflecting the estimation error of $X_{n}$ due to the quantization. Assume that the information of $Y_{n}$ is contained in the quantized block $Y_{n-i_{1}}^{n+i_{2}}$ denoted by $Q_{n+i_{2}}$ for $i_{1}, i_{2} \geq 0$. Let $\hat{\Pi}_{n}=$ $\left(\hat{\pi}_{n}\left(x_{1}\right), \cdots, \hat{\pi}_{n}\left(x_{S}\right)\right)$ be the conditional PMF of $X_{n}$ given $\left\{\Pi_{0}, R_{n-i_{1}}, Q_{n+i_{2}}\right\}$, and let $\tilde{\Pi}_{n}$ be the conditional PMF of $X_{n}$ given $\left\{\Pi_{0}, R_{n-i_{1}}, Y_{n-i_{1}}^{n+i_{2}}\right\}$. Note that full information about $Y_{n}$ is used in computing $\tilde{\Pi}_{n}$. Then we define $J^{e}(n)=\rho\left(\hat{\Pi}_{n}, \tilde{\Pi}_{n}\right)$, where $\rho(\cdot, \cdot)$ is some metric on the space of probabilities on $\mathcal{X}$. In this paper the $l_{1}$ metric on $\mathbb{R}^{S}$ is used. Other metrics such as the Kullback-Liebler divergence can also be used.

The joint quantization and estimation problem is to find $\omega^{*} \in \Omega_{1}$, such that

$$
J\left(\Pi_{0}, \omega^{*}\right)=\min _{\omega \in \Omega_{1}} J\left(\Pi_{0}, \omega\right)=: V\left(\Pi_{0}\right) .
$$

\subsection{The dynamic programming equation}

The joint quantization and estimation problem can be recast as a stochastic control problem and be solved using dynamic programming, as done in [9]. The conditional PMF $\hat{\Pi}_{n}$ is the information state for the new stochastic control problem while the quantization decision $\omega$ is the "control". One of the differences between this work and [9] is that only sequential quantization is considered in the latter.

As standard in dynamic programming, one can first define a sequence of joint quantization and estimation problems. For $1 \leq i \leq N$, let

$$
J_{i}\left(\Pi_{i-1}, \omega_{i}\right)=E\left[\sum_{n=i}^{N} J^{q}(n)+\lambda_{d} J^{d}(n)+\lambda_{e} J^{e}(n)\right],
$$

and $V_{i}\left(\Pi_{i-1}\right)=\min _{\omega_{i} \in \Omega_{i}} J_{i}\left(\Pi_{i-1}, \omega_{i}\right)$, where $\Omega_{i}$ is the space of admissible quantization decisions for the time period $[i, N]$, and $\Pi_{i-1}$ is the a priori PMF for $X_{i-1}$, i.e., the initial condition for the $i$-th problem. Clearly for $i=1$, we recover the original problem formulated in the previous subsection.

Denote by $\Theta_{j}$ the space of quantization (encoding) schemes for a data block of length $j$, say, $Y_{k}^{k+j-1}$ for $k \geq 1$. There are $M^{j}$ possible outcomes for $Y_{k}^{k+j-1}$, so each $\mathcal{Q} \in \Theta_{j}$ partitions these $M^{j}$ outcomes into groups and the group index will carry (compressed) information about $Y_{k}^{k+j-1}$. In this paper we are concerned with the estimation of $X_{n}$ and not the reconstruction of $Y_{n}$; however, considerations of decoding (to the space $\mathcal{Y})$ and the associated compression error can be easily accomodated in the current framework once an appropriate metric is defined on the discrete set $\mathcal{Y}$.
A recursive formula exists for $\hat{\Pi}_{n}$. Assume that for $i \geq 1$, the data block $Y_{i}^{i+j-1}$ of length $j$ is quantized with $\mathcal{Q} \in \Theta_{j}$ and transmitted at time $i+j-1$. Then the conditional marginal PMFs of $X_{i}^{i+j-1}$ can be written in terms of $\hat{\Pi}_{i-1}$ and $\mathcal{Q}\left(Y_{i}^{i+j-1}\right)$ by the Bayes rule:

$$
\left(\begin{array}{c}
\hat{\Pi}_{i} \\
\vdots \\
\hat{\Pi}_{i+j-1}
\end{array}\right)=\left(\begin{array}{c}
f_{j, 1}\left(\hat{\Pi}_{i-1}, \mathcal{Q}\left(Y_{i}^{i+j-1}\right)\right. \\
\vdots \\
f_{j, j}\left(\hat{\Pi}_{i-1}, \mathcal{Q}\left(Y_{i}^{i+j-1}\right)\right.
\end{array}\right)
$$

for some functions $\left\{f_{j, 1}, \cdots, f_{j, j}\right\}=: f_{j}$. The specific forms of $f_{j}$ for $j=1,2$ can be found in [14].

Proposition 2.1 The value functions $\left\{V_{i}\right\}_{i=1}^{N}$ satisfy:

$$
\begin{aligned}
& V_{N}\left(\Pi_{N-1}\right)=1+\min _{\mathcal{Q} \in \Theta_{1}}\left\{H\left[\mathcal{Q}\left(Y_{N}\right)\right]\right. \\
&\left.+\quad \lambda_{e} E\left[\rho\left(f_{1,1}\left(\Pi_{N-1}, \mathcal{Q}\left(Y_{N}\right)\right), \tilde{\Pi}_{N}\right)\right]\right\}
\end{aligned}
$$

and for $1 \leq i \leq N-1$,

$$
\begin{array}{r}
V_{i}\left(\Pi_{i-1}\right)=1+\min _{j \in\{1,2, \cdots, \min (B, N-i+1)\}}\left\{\frac{j(j-1) \lambda_{d}}{2}\right. \\
+\min _{\mathcal{Q} \in \Theta_{j}}\left\{H\left[\mathcal{Q}\left(Y_{i}^{i+j-1}\right)\right]\right. \\
+\lambda_{e} E\left[\sum_{n=i}^{i+j-1} \rho\left(f_{j, n-i+1}\left(\Pi_{i-1}, \mathcal{Q}\left(Y_{i}^{i+j-1}\right)\right), \tilde{\Pi}_{n}\right)\right. \\
\left.\left.\left.+V_{i+j}\left(f_{j, j}\left(\Pi_{i-1}, \mathcal{Q}\left(Y_{i}^{i+j-1}\right)\right)\right)\right]\right\}\right\},
\end{array}
$$

where $V_{N+1}(\cdot) \equiv 0$.

Sketch of proof. For $i=N$, no delay is possible and one quantizes $Y_{N}$ only, which leads to (6). For $i=N-$ 1 , one has the choice to (a) quantize $Y_{N-1}$ alone first and then quantize $Y_{N}$ based on the quantized $Y_{N-1}$, or (b) hold on until $N$ and quantize $Y_{N-1}^{N}$ in one shot. Minimizing over the choice (a) and then the choice (b) leads to (7). Similar arguments can be used to prove the cases for $i<N-2$.

In solving (6) and (7) one obtains the optimal quantization policy for each stage. Concatenating the optimal quantization schemes (with variable block length) yields the optimal quantization decision for the original problem (3).

\section{Numerical Results}

\subsection{Numerical solution of the DP}

To solve the DP equations numerically, one needs to enumerate and compare all partition (encoding) schemes for the finite, discrete sets $\mathcal{Y}^{j}, 1 \leq j \leq B$, where $\mathcal{Y}^{j}$ is the product space of $j$ copies of $\mathcal{Y}$. Each partition for $\mathcal{Y}^{j}$ corresponds to an element of $\Theta_{j}$. For a discrete set $D$, the number of partitions grow rapidly with the cardinality $n_{D}$ of $D$. How to enumerate all 
partitions without repetition is an important issue since repetitions might substantially add to the computational complexity.

An effective method is developed here to eliminate all redundant partitions. The procedure consists of two steps. In the first step a tree-structured algorithm is used to find all the partition patterns. In the second step we list corresponding partitions for each pattern, during which any remaining redundant partition is removed by comparing the "characteristic numbers" of partitions.

By the partition pattern for a (disjoint) partition of the set $D$, we mean a nonincreasing sequence of positive integers where each integer corresponds to the cardinality of one cluster in the partition. For example, if we partition a 7 -element set into 3 clusters, one with 3 elements and the other two with 2 elements each. Then the partition pattern is ( 322 ). A tree is constructed to list all patterns. Each node of the tree has two attributes, "current value" and "remainder". The root node has "current value" $=0$ and "remainder" $=n_{D}$. The root has $n_{D}$ children whose "current value"s are $n_{D}, n_{D}-1, \cdots, 1$, respectively. The "remainder" of each node equals its parent node's "remainder" minus its own "current value". For a nonroot node with "current value" $i_{1}$ and "remainder" $i_{2}$, it will have $i_{0}=\min \left(i_{1}, i_{2}\right)$ children whose "current value"s are $i_{0}, i_{0}-1, \cdots, 1$, respectively. A node is a leaf if its "remainder" is 0 . Every path from the root to a leaf is identified with a partition pattern if one reads off the "current values" of the nodes (except the root) along the path.

Given a pattern, we generate all the corresponding partitions by choosing appropriate numbers of elements from $D$ and putting them into groups. However, if an integer number greater than 1 occurs more than once in a pattern, repetitive enumeration of certain partitions will occur. Such redundancies can be virtually removed using the characteristic numbers defined for partitions (for details, please refer to [14]).

\subsection{Simulation results}

We have conducted calculation and simulation for a two-state, two-output hidden Markov chain. The following parameters have been used: $B=2, N=10$,

$$
\left(a_{i j}\right)=\left[\begin{array}{ll}
0.2 & 0.4 \\
0.8 & 0.6
\end{array}\right],\left(c_{i j}\right)=\left[\begin{array}{ll}
0.3 & 0.7 \\
0.1 & 0.9
\end{array}\right],
$$

By varying the weighting constants $\lambda_{d}$ and $\lambda_{e}$, we compute and store a family of optimal quantization policies. For the initial condition $\Pi_{0}=(0.9,0.1), 50$ sample output trajectories are obtained by simulation. Each quantization policy is applied to these output trajectories, and the average accumulative communication cost $\bar{J}^{q}$, delay $\bar{J}^{d}$, and estimation error $\bar{J}^{e}$ are calculated.

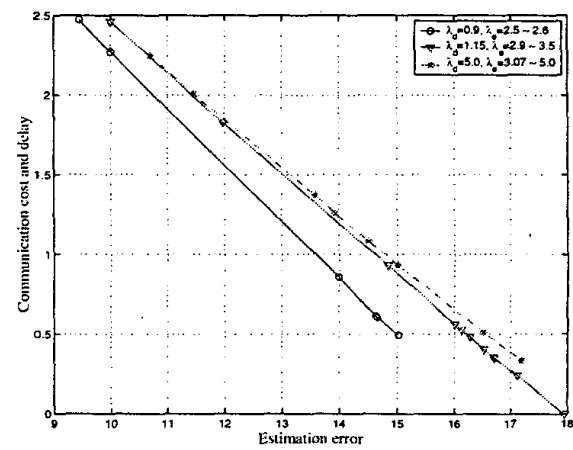

Fig. 2: Weighted combination of communication cost and delay vs. estimation error (points with lower estimation error corresponding to higher $\lambda_{e}$ ).

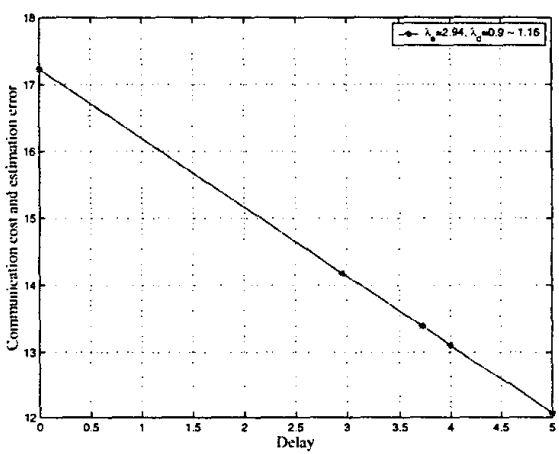

Fig. 3: Weighted combination of communication cost and estimation error vs. delay (points with smaller delay corresponding to higher $\lambda_{d}$ ).

In Fig. 2, each curve shows the variation of combined communication cost and delay vs. the estimation error as $\lambda_{e}$ is changed ( $\lambda_{d}$ is fixed for each curve). The vertical axis is $\bar{J}^{q}+\lambda_{d} \bar{J}^{d}$ and the horizontal axis is $\bar{J}^{e}$. We have also found that (not shown in the figure), for $\lambda_{d}=5.0$, the accumulative delay cost $=0$ (exclusively sequential quantization); for $\lambda_{d}=0.9$, the accumulative delay cost $=5.0$ (exclusively block-coding of length 2); while for $\lambda_{d}=1.15$, variable-length block coding is observed. Fig. 3 shows the variation of combined communication cost and estimation error vs. the delay as $\lambda_{d}$ is changed, where the vertical axis is $\bar{J}^{q}+\lambda_{e} \bar{J}^{e}$ and the horizontal axis is $\bar{J}^{d}$. From the figures we see that jointly optimal quantization decisions vary with the weighting coefficients, and by appropriately choosing these coefficients we can achieve the desired tradeoffs among different objectives. 


\section{Joint Quantization and Control}

\subsection{Problem formulation}

Recall Fig. 1. We now restrict ourselves to sequential vector quantization of $Y_{n}$, i.e., $Y_{n}$ is quantized and transmitted at every $n$. Denote the quantization scheme at $n$ as $\mathcal{Q}_{n} \in \Theta_{1}$, and let $q_{n}=\mathcal{Q}_{n}\left(Y_{n}\right)$. Let $\delta_{n}=\left(\mathcal{Q}_{n}, U_{n}\right)$. We call $\left\{\delta_{n}\right\}$ jointly admissible if $U_{n}$ depends only on $\left\{\Pi_{0}, q_{0}^{n}, U_{0}^{n-1}\right\}$, and $\mathcal{Q}_{n}$ depends only on $\left\{\Pi_{0}, q_{0}^{n-1}, U_{0}^{n-1}\right\}$. Fix $N \geq 1$, and let $\Delta_{0}$ be the space of jointly admissible quantization schemes and controls for the time period $[0, N-1]$. Given $\Pi_{0}$ for $X_{0}$ and $\xi_{0} \in \Delta_{0}$, the cost function is defined as

$$
J\left(\Pi_{0}, \xi_{0}\right)=E\left[\sum_{n=0}^{N-1} \lambda_{q} J^{q}(n)+J^{p}(n)\right],
$$

and the value function is defined as

$$
V\left(\Pi_{0}\right)=\min _{\xi_{0} \in \Delta_{0}} J\left(\Pi_{0}, \xi_{0}\right),
$$

where $\lambda_{q} \geq 0$ is a weighting constant, and $J^{q}(n)$ and $J^{p}(n)$ are the costs relating to communication and performance at time $n$, respectively. $J^{q}(n)$ takes the form in Section 2 if the entropy coding for $q_{n}$ is used, and $J^{q}(n)=\log _{2}\left|q_{n}\right|$ if a plain coding for $q_{n}$ is used, where $\left|q_{n}\right|$ denotes the number of possible outcomes of $q_{n}$. In the following we let $J^{q}(n)=h\left(q_{n}\right)$ for some suitable function $h(\cdot)$. Assume that $J^{p}(n)$ depends on the state and the control, $J^{p}(n)=g_{n}\left(X_{n}, U_{n}\right)$, for some function $g_{n}(\cdot, \cdot)$.

\subsection{The dynamic programming equation}

Denote by $\bar{\Pi}_{i}=\left\{\bar{\pi}_{i}\left(x_{1}\right), \cdots, \bar{\pi}_{i}\left(x_{S}\right)\right\}$ the conditional PMF of $X_{i}$ given $\Pi_{0}, q_{0}^{i-1}$ (and the corresponding quantization schemes), and $U_{0}^{i-1}$. A recursive formula for $\bar{\Pi}_{i}$ can be derived:

$$
\bar{\Pi}_{i+1}=\bar{f}\left(\bar{\Pi}_{i}, q_{i}, U_{i}\right),
$$

for some function $\bar{f}(\cdot, \cdot, \cdot)$. To be specific, for $1 \leq l \leq S$,

$$
\bar{\pi}_{i+1}\left(x_{l}\right)=\frac{\sum_{s=1}^{S} a_{l s}\left(U_{i}\right) \bar{\pi}_{i}\left(x_{s}\right)\left(\sum_{m=1}^{M} 1\left(\mathcal{Q}_{i}\left(y_{m}\right)=q_{i}\right) c_{s m}\right)}{\sum_{t=1}^{S} \bar{\pi}_{i}\left(x_{t}\right)\left(\sum_{m=1}^{M} 1\left(\mathcal{Q}_{i}\left(y_{m}\right)=q_{i}\right) c_{t m}\right)},
$$

where $1(\cdot)$ denotes the indicator function. For $0 \leq$ $i \leq N-1$, let $\Delta_{i}$ be the space of jointly admissible quantization schemes and controls for the time period $[i, N-1]$. For $\xi_{i} \in \Delta_{i}$, define

$$
J_{i}\left(\Pi_{i}, \xi_{i}\right)=E\left[\sum_{n=i}^{N-1} \lambda_{q} h\left(\mathcal{Q}_{n}\left(Y_{n}\right)\right)+g_{n}\left(X_{n}, U_{n}\right)\right],
$$

and $V_{i}\left(\Pi_{i}\right)=\min _{\xi_{i} \in \Delta_{i}} J_{i}\left(\Pi_{i}, \xi_{i}\right)$, where $\Pi_{i}$ is the initial condition for the $i$-th problem. Following a standard DP path one can prove:
Proposition 4.1 For $\mathcal{Q} \in \Theta_{1}$, denote by $\mathcal{A}_{\mathcal{Q}}$ the space of functions mapping the range of $\mathcal{Q}$ to $\mathcal{U}$. The value functions $\left\{V_{i}\right\}_{i=0}^{N-1}$ satisfy:

$$
\begin{array}{r}
V_{N-1}\left(\Pi_{N-1}\right)=\min _{\mathcal{Q}_{N-1} \in \Theta_{1} \alpha_{N-1} \in \mathcal{A}_{\mathcal{E}_{N-1}}} E\left[\lambda_{q} h\left(q_{N-1}\right)\right. \\
\left.+E\left[g_{N-1}\left(X_{N-1}, \alpha_{N-1}\left(q_{N-1}\right)\right) \mid q_{N-1}\right]\right]
\end{array}
$$

where $q_{N-1}=\mathcal{Q}_{N-1}\left(Y_{N-1}\right)$, and for $0 \leq i \leq N-2$,

$$
\begin{array}{r}
V_{i}\left(\Pi_{i}\right)=\min _{\mathcal{Q}_{i} \in \Theta_{1}} \min _{\alpha_{i} \in \mathcal{A}_{\mathcal{Q}_{i}}} E\left[\lambda_{q} h\left(q_{i}\right)+\right. \\
\left.E\left[g_{i}\left(X_{i}, \alpha_{i}\left(q_{i}\right)\right) \mid q_{i}\right]+V_{i+1}\left(\bar{f}\left(\Pi_{i}, q_{i}, \alpha_{i}\left(q_{i}\right)\right)\right)\right],
\end{array}
$$

where $q_{i}=\mathcal{Q}_{i}\left(Y_{i}\right)$. From the solutions $\left\{\left(\mathcal{Q}_{i}^{*}, \alpha_{i}^{*}\right)\right\}_{i=0}^{N-1}$ to (12) and (13) one can construct the jointly optimal quantization and control schemes.

Similar to the joint quantization/estimation problem, the separation principle holds. The conditional PMF $\bar{\Pi}_{n}$ of $X_{n}$ is the information state, and the optimal quantization/control scheme depends only on $\bar{\Pi}_{n}$.

\subsection{An example problem}

We take the machine repair problem from [15] (pp. 190) as an example. A machine can be in one of two states denoted by $P$ (Proper state) and $\bar{P}$ (Improper state). If the machine starts in $P$ and runs for one period, its new state will remain $P$ with probability $\frac{2}{3}$, and if it starts in $\bar{P}$, it will remain in $\bar{P}$ with probability 1 . At the beginning of each time period, one takes an inspection to help determine the machine's state. There are two possible inspection outcomes denoted $G($ Good) and $B$ $(B a d)$. If the machine is in $P$, the inspection outcome is $G$ with probability $\frac{3}{4}$; if the machine is in $\bar{P}$, the inspection outcome is $B$ with probability $\frac{3}{4}$. After each inspection, one of two possible actions can be taken, $C$ (operate the machine for one period) or $S$ (stop the machine and perform maintenance, then operate the machine for one period). The running cost for one period is 2 units if the machine is in state $\bar{P}$, and is 0 if it is in $P$. The action $S$ makes sure that the machine is in $P$ but it costs 1 unit.

To relate this problem to the joint quantization/control problem discussed earlier, we assume that the inspection outcome needs to be sent to a remote site for action decision. A plain coding scheme requires one bit to send the information $G$ or $B$. The only other quantization scheme is to cluster $G$ and $B$, which requires 0 bit for communication. $\lambda_{q}$ now carries an interpretation of communication cost per bit.

Given the a priori PMF of the machine state at time 0 , the problem is to decide at the beginning of each time period whether to communicate the inspection outcome and what action to take based on the received information, so that the total cost is minimized. One can show that the value function of this problem is concave and 


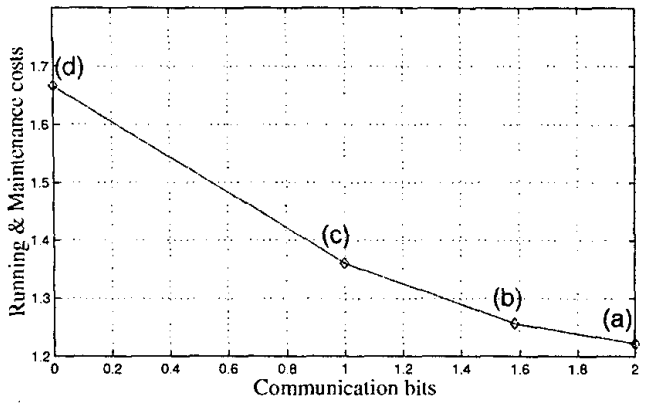

Fig. 4: Running and maintenance costs vs. communication bits for jointly optimal strategies.

piecewise linear. We have obtained the explicit solution for $N=2$. Take $\operatorname{Pr}\left[X_{0}=P\right]=\frac{2}{3}$. Then one of the following four joint quantization/control strategies becomes optimal depending on the value $\lambda_{q}$ :

(a) $\left[\lambda_{q} \leq \frac{1}{12}\right]$ At time 0 , send the inspection outcome $Y_{0}$, and let $U_{0}=C(S$, resp. $)$ if $Y_{0}=G(B$, resp. $)$; At time 1 , send $Y_{1}$, and let $U_{1}=C(S$, resp. $)$ if $Y_{1}=$ $G(B$, resp.);

(b) $\left[\frac{1}{12}<\lambda_{q} \leq \frac{5}{28}\right]$ At time 0 , send $Y_{0}$, and let $U_{0}=$ $C(S$, resp. $)$ if $Y_{0}=G(B$, resp. $)$; At time 1 , if $Y_{0}=G$, send $Y_{1}$ and let $U_{1}=C\left(S\right.$, resp.) if $Y_{1}=G(B$, resp. $)$, and if $Y_{0}=B$, let $U_{1}=C$ without sending $Y_{1}$;

(c) $\left[\frac{5}{28} \leq \lambda_{q}<\frac{11}{36}\right]$ At time 0 , send $Y_{0}$, and let $U_{0}=$ $C(S$, resp. $)$ if $Y_{0}=G(B$, resp. $)$; At time 1 , let $U_{1}=C$ without sending $Y_{1}$;

(d) $\left[\lambda_{q} \geq \frac{11}{36}\right]$ At time 0 , let $U_{0}=C$ without sending $Y_{0}$; at time 1 , let $U_{1}=S$. without sending $Y_{1}$.

In Fig. 4 the expected accumulative running and maintenance cost vs. the expected bits of communication for these four strategies is shown. The thresholds of $\lambda_{q}$ for switching of the optimal strategy correspond to the negative slopes of the line segments connecting the neighboring points in Fig. 4. Hence when the communication cost per bit increases, the optimal strategy tends not to transmit the inspection outcome.

\section{Conclusions}

In this paper the problem of joint quantization, estimation, and control of a hidden Markov chain has been studied. We first investigated the joint quantization and estimation problem, where vector quantization with variable-block length was considered. Then the joint quantization and control problem was formulated and solved. The common theme for these two problems is that a weighted combination of different costs is minimized. By varying the weighting coefficients, one can obtain a family of optimal quantization/control schemes that reflect different tradeoff strategies. Simulation and a simple example have been used to illustrate the results.
The framework presented in this paper can be extended to continuous-range systems. The case of noisy communication can also be incorporated. Finally we note that, as a drawback of the approach, the "curse of dimensionality" of DP applies when the number of states gets large.

\section{References}

[1] W. S. Wang and R. W. Brockett, "Systems with finite communication bandwidth constraints - II: stabilization with limited information feedback," IEEE Trans. Automat. Control, vol. 44, no. 5, pp. 1049-1053, 1999.

[2] N. Elia and S. K. Mitter, "Stabilization of linear systems with limited information," IEEE Trans. Automat. Control, vol. 46, no. 9, pp. 1384-1400, 2001.

[3] J. Baillieul, "Feedback coding for information-based control: operating near the data-rate limit," in Proceedings of the 41st IEEE Conference on Decision and Control, Las Vegas, NV, 2002, pp. 3229-3236.

[4] V. Borkar and S. Mitter, "LQG control with communication constraints," in Communications, Computation, Control, and Signal Processing: A Tribute to Thomas Kailath, A. Paulraj, V. Roychowdhury, and C. Schaper, Eds. 1997, pp. 365-373, Kluwer Academic Publishers.

[5] W. S. Wong and R. W. Brockett, "Systems with finite communication bandwidth constraints - Part I: state estimation problems," IEEE Trans. Automat. Control, vol. 42, no. 9, pp. 1294-1299, 1997.

[6] S. Tatikonda, Control under Communication Constraints, Ph.D. thesis, M.I.T., 2000.

[7] V. S. Borkar, S. K. Mitter, and S. Tatikonda, "Markov control problems under communication constraints," Communications in Information and Systems, vol. 1, no. 1, pp. 15-32, 2001.

[8] R. J. Elliott, L Aggoun, and J. B. Moore, Hidden Markov Models: Estimation and Control, Springer-Verlag, 1995.

[9] V. S. Borkar, S. K. Mitter, and S. Tatikonda, "Optimal sequential vector quantization of Markov sources," SIAM J. Control Optim., vol. 40, no. 1, pp. 135-148, 2001. [10] P. A. Chou, T. Lookabaugh, and R. M. Gray, "Entropy-constrained vector quantization," IEEE Transactions on Acoustics, Speech, and Signal Processing, vol. 37, no. 1, pp. 31-42, 1989.

[11] J. S. Baras and S. Dey, "Combined compression and classification with learning vector quantization," IEEE Trans. Inform. Theory, vol. 45, no. 6, pp. 1911-1920, 1999. [12] H. S. Witsenhausen, "Separation of estimation and control for discrete time systems," Proceedings of the IEEE, vol. 59 , no. 11 , pp. 1557-1566, 1971.

[13] T. M. Cover and J. A. Thomas, Elements of Information Theory, John Wiley \& Sons, Inc, New York, 1991.

[14] J. S. Baras, X. Tan, and W. Xi, "Jointly optimal quantization, estimation, and control of hidden Markov chains," Tech. Rep. 2003-15, Institute for Systems Research, University of Maryland, 2003.

[15] D. P. Bertsekas, Dynamic Programming and Optimal Control, Athena Scientific, Belmont, MA, 1995. 